\title{
Multiple foreign bodies causing an orocutaneous fistula of the cheek
}

Woo Ju Kim,

Woo Seob Kim,

Han Koo Kim,

Tae Hui Bae

Department of Plastic and Reconstructive Surgery, Chung-Ang University Hospital, Chung-Ang University College of Medicine, Seoul, Korea
Foreign bodies impacted in the maxillofacial region are often a diagnostic challenge. They can be a source of chronic inflammatory reactions and infections leading to the formation of an orocutaneous fistula. Such orocutaneous fistulas cause significant morbidity in most patients, eventually requiring surgery. Recently, we encountered a very rare case of an orocutaneous fistula caused by multiple foreign bodies in the cheek. Precise removal of the foreign bodies was required, and a double-sided anterolateral thigh free flap was used to reconstruct the defect. Surgeons should be aware of the complications of multiple foreign bodies and should be able to diagnose these on careful clinical examination.

Keywords: Orocutaneous fistula / Foreign body / Flap

\section{INTRODUCTION}

The introduction of a foreign body into the orofacial region may be accidental, iatrogenic, or deliberate [1]. Foreign bodies embedded in the oral and maxillofacial region are not unusual. However, the detection of these foreign bodies inside the soft tissue is very difficult owing to the induction of granuloma formation around them. Additionally, some foreign bodies can be a source of chronic inflammatory reactions and infections leading to the formation of an orocutaneous fistula in the impacted area [2].

Orocutaneous fistulas usually occur after surgeries for head and neck malignancies and particularly with radiotherapy. Once formed, most patients experience significant morbidity including extended hospital stays, difficulty eating, and pain. Current treatments for orocutaneous fistulas include aggressive wound care, eradication of sepsis, and the maintenance of overall nutrition via gastrostomy, jejunostomy, or nasogastric tubes [3]. However, due

\section{Correspondence: Tae Hui Bae}

Department of Plastic and Reconstructive Surgery, Chung-Ang University Hospital, 102 Heukseok-ro, Dongjak-gu, Seoul 06973, Korea

E-mail: psbth@cau.ac.kr

Received March 23, 2018 / Revised March 23, 2018 / Accepted May 7, 2018 to slow wound healing and a reduced quality of life, a surgical approach with flap reconstruction is often required eventually.

Recently, we experienced a rare case of an orocutaneous fistula caused by multiple foreign bodies in the cheek, reconstructed with an anterolateral thigh (ALT) free flap. To best of our knowledge, this is the first case report in the current literature of an orocutaneous fistula induced by multiple foreign bodies.

\section{CASE REPORT}

A 75-year-old male patient visited the plastic and reconstructive surgery department for recurrent swelling, pain, and a cutaneous tract of the right cheek (Fig. 1). Based on the patient's history, he had had a $1 \mathrm{~cm} \times 2 \mathrm{~cm}$ mass in the right buccal area and had undergone excision 2 years previously in China. After the operation, for the purpose of prevention, more than 20 foreign objects (linear metal needles) were inserted at the operational site (Fig. 2). From that point, a small opening on the right oral mucosa appeared and slowly progressed to connect to the external cheek over a period of nearly 2 years.

The first fistula repair included a mucosa advancement flap on 
the inside, while a local rotation flap was used on the outside. During the operation, 22 linear metal needles were removed (Fig. 3). However, a month after the first operation, the fistula recurred in the same location with an even bigger opening, probably due to poor oral hygiene (Fig. 4). Immediately, a nasogastric tube was inserted, and several debridement procedures were carried out. An ALT free flap with a de-epithelized double-sided design was used for the second fistula repair (Fig. 5).

After a subsequent hematoma removal, the postoperative result was favorable without any major complication or recurrence.
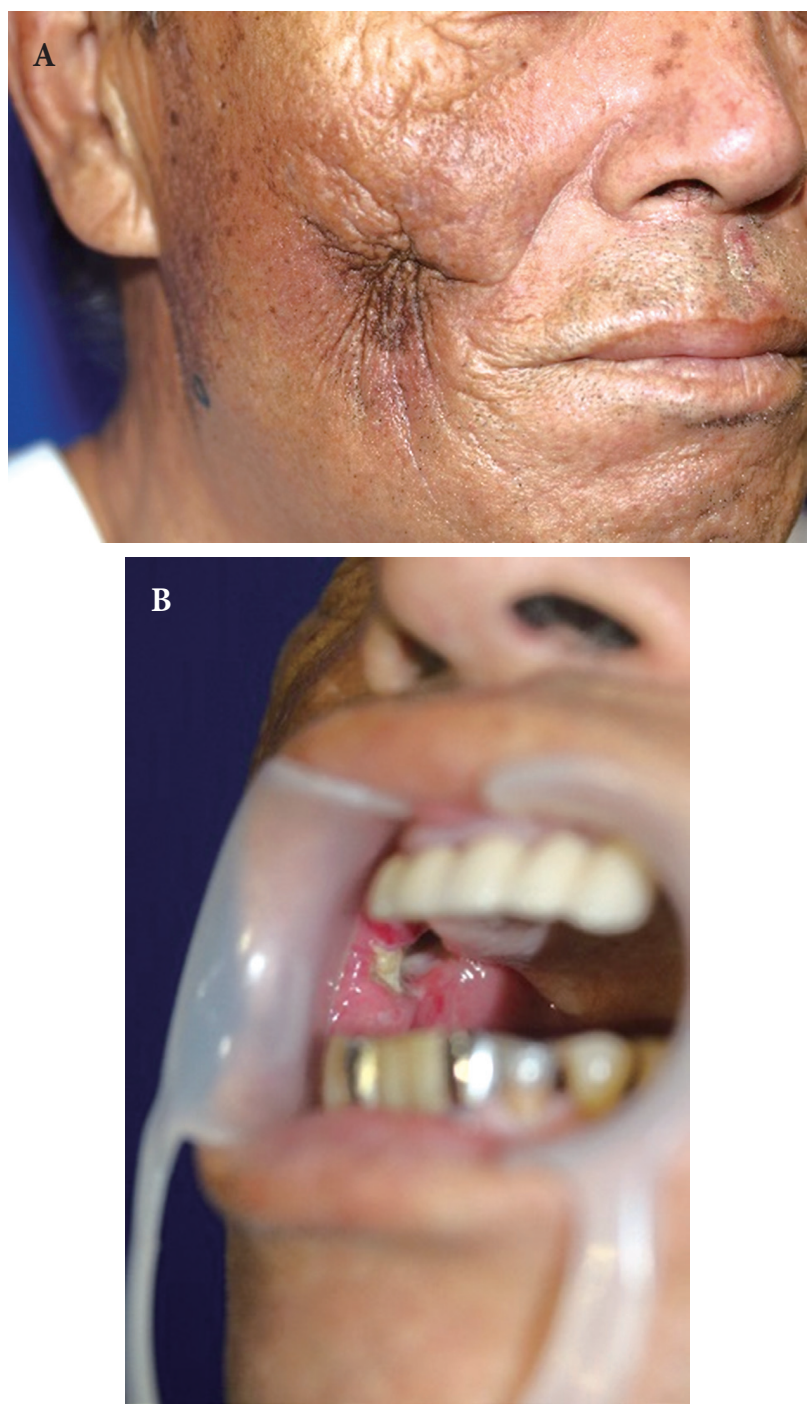

Fig. 1. A 75-year-old male patient presented with an orocutaneous fistula on the right cheek. (A) Profile view. (B) Oral view showing the internal opening of the orocutaneous fistula.
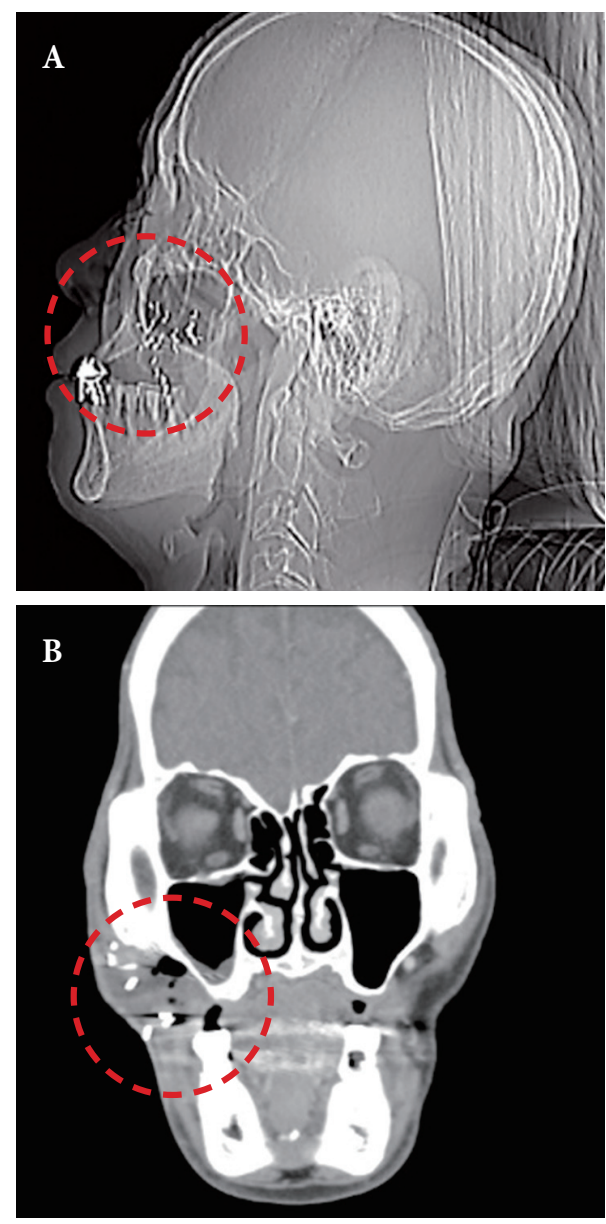

Fig. 2. Preoperative radiographic findings showing impacted foreign bodies (red circled areas). (A) Plain radiography, lateral view. (B) Computed tomography, coronal view.

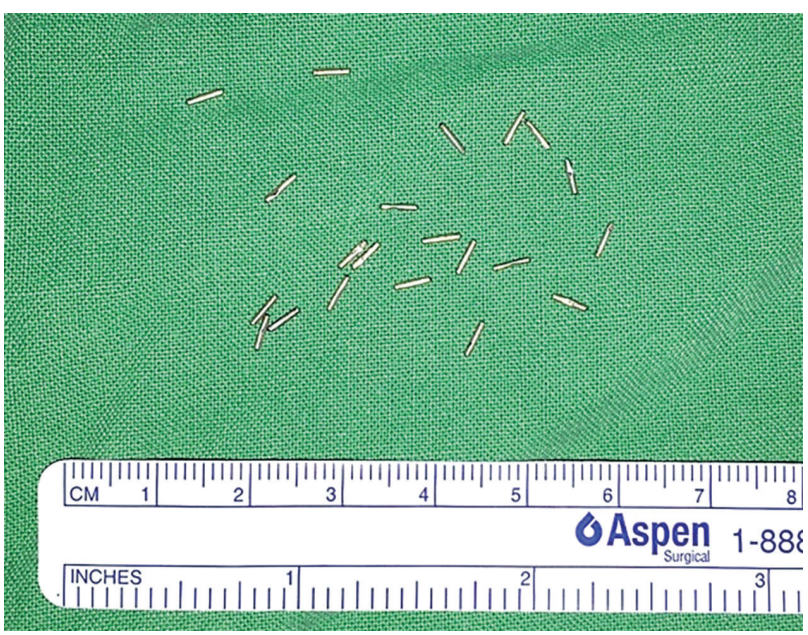

Fig. 3. Retrieved foreign bodies during the operation: 22 linear metal needles (each needle was $4 \mathrm{~mm}$ in length). 


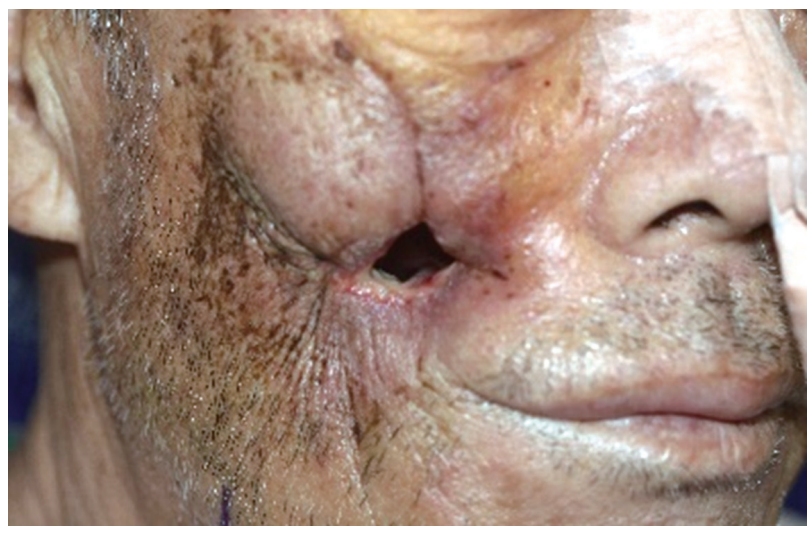

Fig. 4. Recurrent orocutaneous fistula with an even bigger opening at the same location.
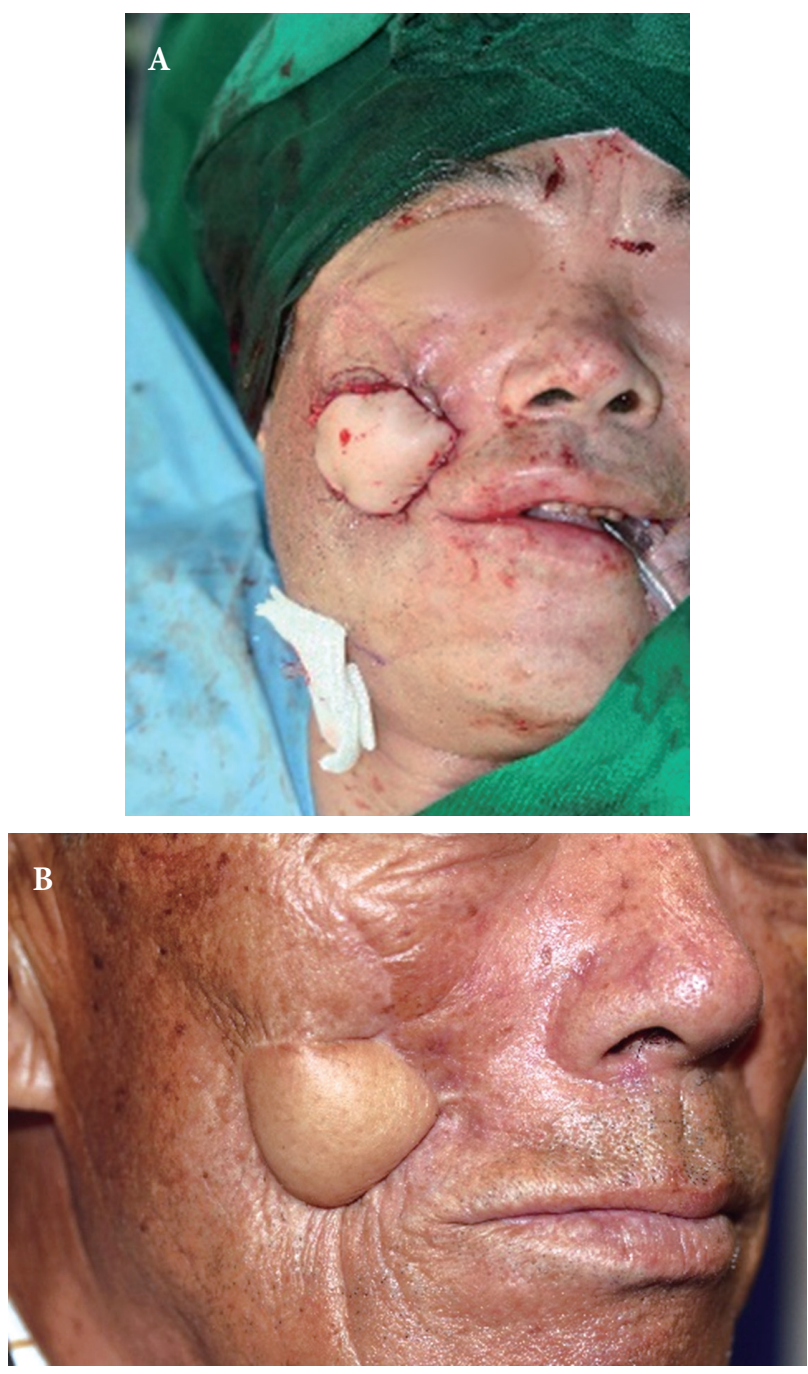

Fig. 5. Postoperative view with a double-sided anterolateral thigh free flap. (A) Immediate postoperative view. (B) Two-year postoperative view.

\section{DISCUSSION}

Foreign bodies impacted in the maxillofacial region are often a diagnostic challenge because of many factors such as the size of the object, the difficult access, and a close anatomic relationship of the foreign bodies to vital structures [1]. They can induce an intense inflammatory reaction in the surrounding tissues and may cause a secondary infection so the patient may present with recurrent, non-healing sinuses in the orofacial region, similar to those in our case.

In general, these foreign bodies in the orofacial area should be removed. Indications for removal include neurovascular compromise, evidence of infection, cosmetic deformity, functional impairment, chronic pain, and the patient's request [4]. To retrieve the foreign bodies from such a complex anatomical region, accurate imaging is the most important feature. Among the various imaging modalities, computed tomography (CT) is the modality of choice for the detection of most foreign bodies, as in our case. However, if the foreign body consists of wooden material, the role of the CT scan is limited. Earlier reports suggest that ultrasound is a superior imaging modality to CT scanning and magnetic resonance imaging for the detection of vegetative foreign bodies in the soft tissues [2].

Various reconstructive strategies have been reported in the literature, including single-layered fasciocutaneous island flaps, local flaps, or free flaps. Negative pressure wound therapy is another recent trend, but further evidence from larger studies needs to be collected. In spite of these methods, the recurrence rates remain high, reportedly up to $35 \%$ when using a pectoralis major muscle flap [5]. Therefore, we decided to use an alternative option to recreate an inner lining and an outer cover in a single stage with a relatively low recurrence rate: the double-sided ALT free flap. According to several studies, double-layer techniques have produced successful results for orocutaneous fistulas and pharyngocutaneous fistula reconstructions [6].

According to the current literature, a definite treatment protocol for foreign bodies in the maxillofacial area has not been established. In particular, orocutaneous fistulas caused by foreign bodies are especially difficult to diagnose and manage. Surgeons must 
perform careful clinical examination with a precise review of the patient's history, such as trauma and dental history [7]. Detection of the foreign bodies inside the soft tissue must be performed with various modalities for accurate localization. Aggressive wound care with surgical removal of foreign bodies is essential to manage foreign bodies induced by orocutaneous fistulas, and the remaining defects often require surgical reconstruction [8].

To best of our knowledge, this is a very rare case of an orocutaneous fistula caused by multiple foreign bodies in the maxillofacial area. We report this case to highlight that surgeons should be aware of the complications of multiple foreign bodies in the maxillofacial area and should be able to diagnose them with adequate imaging modalities. Additionally, precise removal of foreign bodies and appropriate surgical reconstruction should be planned to eliminate recurrence.

\section{CONFLICT OF INTEREST}

No potential conflict of interest relevant to this article was reported.

\section{PATIENT CONSENT}

The patients provided written informed consent for the publication and the use of their images.

\section{REFERENCES}

1. Bede SY, Ahmed FT. Management of retained foreign bodies in missile injuries of the maxillofacial region. J Craniofac Surg 2011;22:1440-4.

2. Auluck A, Behanan AG, Pai KM, Shetty C. Recurrent sinus of the cheek due to a retained foreign body: report of an unusual case. $\mathrm{Br}$ Dent J 2005;198:337-9.

3. Raman R, Ariayanayagam C. Closure of orocutaneous and pharyngocutaneous fistulas. Plast Reconstr Surg 1987;79:310.

4. Naude GP, Bongard FS, Demetriades D. Trauma secrets. Philadelphia: Hanley \& Belfus; 2003

5. McLean JN, Nicholas C, Duggal P, Chen A, Grist WG, Losken A, et al. Surgical management of pharyngocutaneous fistula after total laryngectomy. Ann Plast Surg 2012;68:442-5.

6. Sadigh PL, Wu CJ, Feng WJ, Hsieh CH, Jeng SF. New double-layer design for 1-stage repair of orocutaneous and pharyngocutaneous fistulae in patients with postoperative irradiated head and neck cancer. Head Neck 2016;38 Suppl 1:E353-9.

7. Sammut S, Malden N, Lopes V. Facial cutaneous sinuses of dental origin: a diagnostic challenge. Br Dent J 2013;215:555-8.

8. Cohen M, Marschall MA, Greager J 3rd. Early, aggressive management of postoperative oropharyngocutaneous fistulas. Plast Reconstr Surg 1992;89:56-61. 